\title{
The Effect of Loading Ratios and Electric Field on Charge Dynamics in Silica-based Polyethylene Nanocomposites
}

\author{
Yan Wang, Dayuan Qiang, Zhiqiang Xu, George Chen, and Alun Vaughan \\ School of Electronics and Computer Science, University of Southampton, Southampton, United \\ Kingdom \\ E-mail: yw14g13@soton.ac.uk
}

\begin{abstract}
Nanodielectrics have been expected to improve the electrical performance and considered as dielectrics for the future. It has been recognized that the electrical performance is close related to charge dynamics in the dielectrics material. However, the mechanism of charge dynamics in the interphase of nanodielectrics has not been fully understood, which cause the difficulty in understanding the effect of nanoparticle loading ratios and electric fields applied on the electrical properties. Recently, a model based on the tunneling process with the presence of deep traps has been suggested as one of the conceivable candidates for explaining charge dynamics in nanodielectrics, but the related experiment results are not discussed with tunnelling process. In this paper, the measurements including isothermal surface potential decay and space charge are conducted for the blend polyethylene incorporated with the untreated silica nanocomposites. According to the experimental observation compared with the unfilled blend polyethylene, the electrical properties of nanocomposites with high loading ratios of $2 \mathrm{wt} \%, 5 \mathrm{wt} \%$ and $10 \mathrm{wt} \%$ are worsened such as facilitated space charge accumulation and injection, and faster charge carriers transport. On the other hand, regarding the nanocomposites with the low loading ratios of $0.5 \mathrm{wt} \%$, it was observed that slow transport of charge carriers, and suppressed space charge accumulation and injection. The effect of the lower and higher electric field on the electrical properties of the nanocomposites was similar for the low and high loading ratios. The tunnelling process associated with deep traps can effectively explain these observed phenomena of nanocomposites, it is therefore suggested for further explaining the electrical properties and charge dynamics in the nanodielectrics.
\end{abstract}

\section{INTRODUCTION}

The high voltage direct current (HVDC) transmission system has been in use since the mid-20th century, and is now becoming increasingly important [1]. HVDC cable is the important part of the power transmission, especially for the offshore windfarm. However, one of the primary challenges in the development of HVDC cable is the easy accumulation of space charge in the insulation materials. The formation of space charge can lead to higher local electric field, which may result in insulation materials overstressed locally [2,3]. As a result, the degradation and breakdown of the insulation may be facilitated [4]. Since the nanodielectrics was proposed from 1994 [5], this new generation insulating material is expected to globally improve the electrical properties in contrast to conventional polymeric dielectrics and microcomposites, especially for offering high breakdown strength and suppressing the accumulation of space charge. Nanometer-sized fillers have very high ratios of surface

1. Electronic mail: yw14g13soton.ac.uk. 
area to volume, and the surface of incorporated nanoparticles would form a large interphase with the matrix [5], which is the key to leading globally improved electrical properties [6]. During the next two decades, the inspired experiment results of the $\mathrm{MgO} /$ low density polyethylene (LDPE) nanocomposites were frequently cited [7] because it can effectively suppress the accumulation of space charge and obviously enhance the DC breakdown strength approaching 50\% (around $900 \mathrm{kV} / \mathrm{mm}$ ) on doping just $2 \%$ of this nanoparticle. However, regarding investigations of other nanocomposites filling such as nanosilica, boron nitride and silica nitride et al., an obvious improvement of electrical properties especially for DC breakdown was rarely reported [8-11]. In addition, the reported suppression of space charge can be only observed for the nanocomposites loaded with low loading ratios $[12,13]$ whereas both homocharges and heterocharges injection were observed for the nanocomposites with high loading ratios $[9,10]$. However, this phenomenon was not drawn sufficient attention and explained effectively.

Various hypothetical models of the interphase have been suggested to describe the more specific electrical characteristics and charge dynamics of nanocomposites. For instance, the model proposed by Lewis in 2004 is based on the gradually changing intensity of a material property over several nanometers [14]. Although this model does not describe any physical process at the interfacial area in nanocomposites, it visualises the impact of nanoscale fillers on the macroscopic material. The multi-core model proposed by Tanaka et al. in 2005 further explains the various phenomena exhibited by nanocomposite dielectrics [15]. This model layers the interaction area between an inorganic nanoparticle and a matrix, which mainly consists of a bonded layer, a bound layer, and a loose layer. It effectively explains the partial discharge resistance of polyamide/layered silicate nanocomposites but lacks discussion about the mechanism of the observed space charge phenomena. In 2008, Takada et al. proposed the trapping model based on the concept of 'induced dipole polarisation' [12]; This model indicates that the suppression mechanism of charge accumulation in LDPE/MgO nanocomposites stems from deep traps induced by a small amount of nanoparticles, but it does not explain the enhanced charge transport and accumulation in nanocomposites with high nanoparticle loading ratios. Charge traps is frequently used to explain the space charge dynamics in both nanocomposites and conventional polymer [3,16-18]. Since the traps will affect the charge carrier trapping, detrapping, transportation and recombination processes [19], the energy level (depth), number and distribution of charge traps are crucial to space charge dynamics in polymeric materials. Based on the energy level, the traps can be broadly classified into two types [20], shallow and deep trap. Regarding quantity and space, the nature or the treatment of the materials will affect the trap density and the trap distribution [20]. Moreover, deep traps induced by nanoparticles into the material system is experimentally supported by the following experimental results: (i) a thermally stimulated current (TSC) peak shifted towards a higher temperature following the introduction of nanoparticles [6,21]; (ii) low mobility for charge carriers [22] has been observed in nanocomposites; (iii)the 
deeply trapped charge carriers in the sub-surface region of nanocomposites is experimentally verified as the main reason causing the observed space charge suppression [23].

As the presence of deep traps in nanocomposites is confirmed, the following issue is how to employ deep traps to explain the observed charge dynamics as reported [9,24,25]. The function of deep traps is to capture the charge carriers and slow the transport of them. However, it is inconsistent with the reported space charge accumulation in the nanocomposites with high loading ratios as mentioned previously, and the main issue of causing these phenomena is due to effect of different loading ratios and electric fields. The relevant results obtained may be highly expected since the similar research was conducted since 2012 [10], but an effective explanation of charge transport in nanocomposites with high loading ratios is still missing as an important part of investigation of nanodielectrics. In this paper, the effect of the nanoparticle loading ratios and electric fields applied on the charge dynamics of silica based blend polyethylene nanocomposites are discussed. The studied nanocomposite has shown homogeneous dispersion and consistency of electrical properties [23]. The main aims of this paper are first to show the different behaviour of charge dynamics according to the loading ratios of nanosilica and applied electric fields. Secondly, since the previous models cannot fully explain the obtained results, the tunneling model associated with deep traps [16,26], which will be introduced in details later, is suggested to explain the observed charge dynamics in the nanocomposites with high loading ratio associated with the laminated specimen measurements. The isothermal surface potential decay (ISPD) measurement $[3,27]$ is used in this research for providing information about the characteristics of traps in the nanocomposites associated with spatial charge profile measurements of pulsed electro-acoustic (PEA) method [28,29].

\section{EXPERIMENT DETAILS}

\section{A. Specimen Preparation and Characterization}

The specimen used in this study is nanosilica based blend polyethylene. The host polymer used was $20 \mathrm{wt} \%$ high-density polyethylene (HDPE) obtained from Rigidex HD5813A BP Chemical blend with $80 \mathrm{wt} \%$ low density-polyethylene (LDPE) obtained from LD100BW ExxonMobil Chemicals. The control group without nanofillers incorporated is referred as BPE. Nanosilica used in this study was product of Sigma-Aldrich. The range of its diameters is from $10 \mathrm{~nm}$ to $20 \mathrm{~nm}$. Four weight percentages including $0.5 \mathrm{wt} \%, 2 \mathrm{wt} \%, 5 \mathrm{wt} \%$ and $10 \mathrm{wt} \%$ were used in this investigation. The nanocomposites are referred as ASR. The solvent method was used for manufacture of nanocomposites, which is consistent to the previous research 
$[9,24,25,30]$. First of all, the specific amount of nanosilica was mixed with xylene and sonicated by probe sonication for 1 hour. Afterward, the mixture of polyethylene pellet/nanosilica/xylene stirred by magnetic stirrer with high speed at $150{ }^{\circ} \mathrm{C}$, followed by precipitation into methanol immediately. The obtained precipitated gel was filtered and dried in the vacuum oven at room temperature least 3 days for remove the residual solvent. The specimen was pressed at $160{ }^{\circ} \mathrm{C}$ by the large hydraulic press. For eliminating the impact of different crystallization process on the results of charge dynamics and ensuring the repeatability, the specimen was isothermal crystallized in the oil bath at $115{ }^{\circ} \mathrm{C}[31]$. The thickness of specimen is $\sim 120 \pm 5 \mu \mathrm{m}$, and the area of specimens for surface potential decay was $20 \pm 2 \mathrm{~cm}^{2}$, and for space charge was $10 \pm 2 \mathrm{~cm}^{2}$. The manufacturing procedures were strictly followed from batch to batch.

\section{B. Experiment Configuration of ISPD}

Fig. 1 shows a schematic diagram of the ISPD setup. The distance between the needle electrode and the top of the earth plate was approximately $4.5 \mathrm{~cm}$. The mesh grid electrode was $3 \mathrm{~cm}$ away from the needle electrode. The design of this configuration can primarily ensure the stability of grid voltage and safety of operation. The function of the needle electrode was to generate negative corona discharge with relatively high voltage. The grid electrode served to obtain a uniform charge distribution on the surface of the specimen, because the electric field between the mesh grid and the earth plate was relatively uniform. In addition, the mesh grid voltage determines the initial surface potential. To achieve consistent results, all measurements were carried out and repeated in a controlled environment in which the temperature and relative humidity remained at $23 \pm 3^{\circ} \mathrm{C}$ and $40 \pm 5 \%$ respectively. After negative corona charging, the specimen was moved towards a compact JCI 140 static monitor to observe the isothermal surface charge decay via a rotatable earth plate. The time required to rotate the sample was about 1 second, and the first reading was taken after $5 \pm 1$ seconds due to the delay in data transfer from the monitor to the PC. In this study, the needle voltage was set at $15 \mathrm{kV}$ and the grid voltage was set from $4.8 \mathrm{kV}$ to $7.2 \mathrm{kV}$ with $1.2 \mathrm{kV}$ intervals. The period of corona charging was $180 \mathrm{~s}$ and the decay period was $900 \mathrm{~s}$. This selected time was based on [28], which can ensure the efficiency and clear experiment phenomena to be observed. 


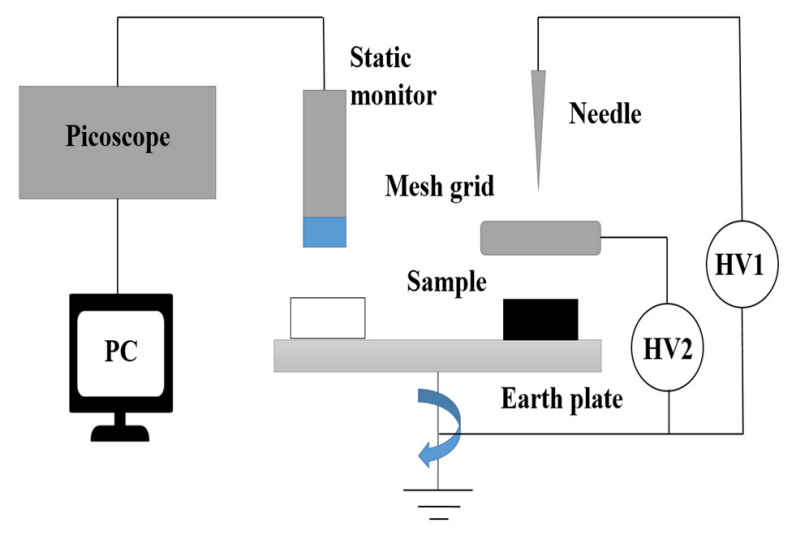

FIG. 1. Isothermal surface potential measurement system

\section{Experiment Configuration of Space Charge}

The PEA system as shown in Fig. 2 has been widely used for space charge profile measurements. The fundamental principle of the PEA system is to use the acoustic pulse which can be generated by the displacement of each locally charged part of insulation materials when interacting with the electric pulse. In the present PEA system, the amplitude of the pulse generator is $600 \mathrm{~V}$ with $5 \mathrm{~ns}$ pulse width. For achieving a better acoustic transmission, a very thin layer of silicone oil was dropped on both sides of the specimen. The semiconducting polymer was used as a top electrode for obtaining better acoustic impedance match and aluminum was used as a bottom electrode. For all the measurements, the calibration was conducted at $10 \mathrm{kV} / \mathrm{mm}$. The tests were monitored over 2 hours for both the volts-on and space charge decay at room temperature.

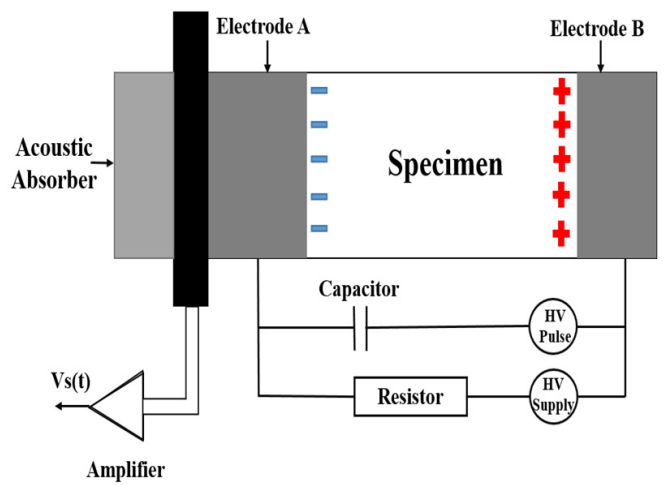

FIG. 2. The schematic diagram of the PEA system 


\section{RESULTS AND ANALYSIS}

\section{A. Space Charge}

The space charge measurement was performed for each specimen at least three times. The vertical dashed lines indicate the location of electrodes. Fig. 3 illustrates the space charge profile of BPE, $0.5 \mathrm{wt} \%$ and $5 \mathrm{wt} \%$ ASR stressed at $50 \mathrm{kV} / \mathrm{mm}$ and $70 \mathrm{kV} / \mathrm{mm}$, which is to reveal the effect of electric field on the charge dynamic. After 120 minutes stressing, the heterocharges are located at the cathode side of the BPE specimen from Fig. 3(a). However, these accumulated heterocharges are not affected significantly as the electric field increases from $50 \mathrm{kV} / \mathrm{mm}$ to $70 \mathrm{kV} / \mathrm{mm}$. With respect to measurement of 0.5 wt $\%$ ASR specimen in Fig. 3 (b), it is observed that space charge is suppressed at $50 \mathrm{kV} / \mathrm{mm}$ whereas a few amount of negative and positive charges can be found in the middle of the bulk at $70 \mathrm{kV} / \mathrm{mm}$. Regarding $5 \mathrm{wt} \%$ nanocomposites, the space charge accumulation is obviously observed at $50 \mathrm{kV} / \mathrm{mm}$. When the applied electric field increases to $70 \mathrm{kV} / \mathrm{mm}$, charge injection becomes strong and more than one positive charge peak can be noticed as shown in Fig. 3(c).
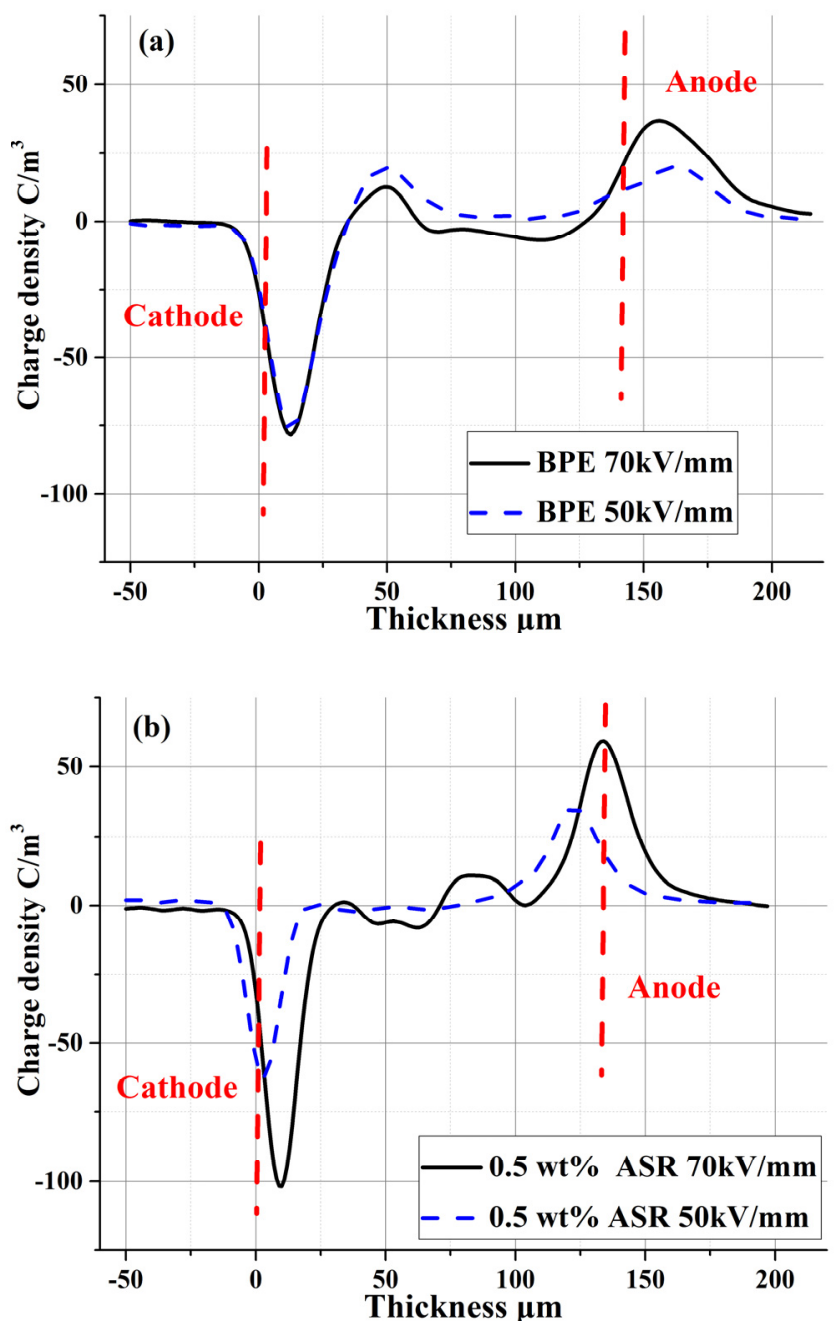


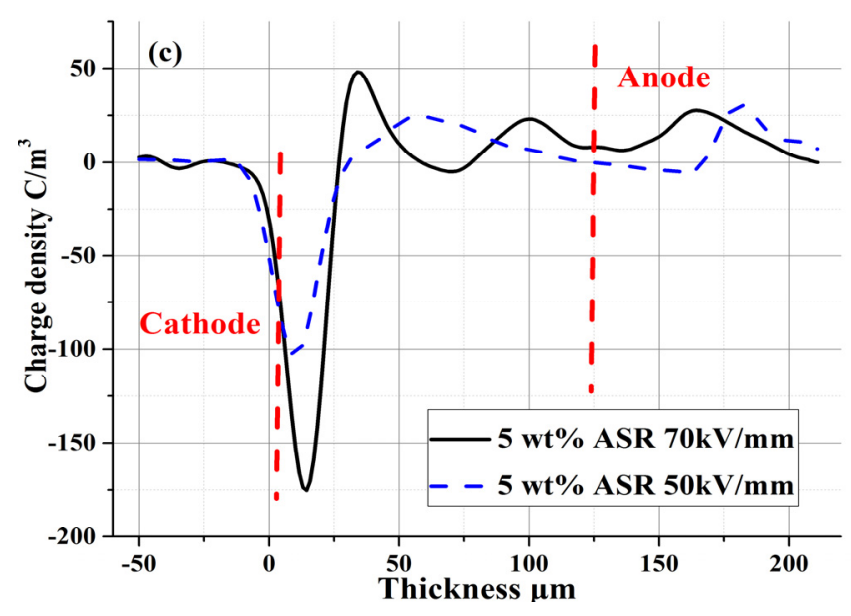

FIG. 3. The space charge profile after 120 minutes poling at different electric field (a) BPE, (b) $0.5 \mathrm{wt} \%$ ASR, (c) $5 \mathrm{wt} \%$ ASR

Fig. 4 shows the effect of different loading ratios on space charge profile at $70 \mathrm{kV} / \mathrm{mm}$. Compared with $0.5 \mathrm{wt} \%$ ASR, $5 \mathrm{wt} \%$ ASR and BPE have more injected charges, as shown in Fig. 4 (a1)-(c1). The heterocharges can be observed for the $5 \mathrm{wt} \%$ ASR and the BPE. Fig. 4 (a1) shows that most of the heterocharges in the BPE are positive, which is partly due to the higher charge carrier injection rate of the semiconducting polymer on the anode, in contrast to the aluminum on the cathode [32]. In addition, considering the neutralisation as a result of the recombination process, even fewer negative charges can be observed in the BPE. The amount of charges in $5 \mathrm{wt} \%$ ASR is more than the BPE, which supports that nanocomposites with relatively higher loading ratios can enhance the formation of space charge. Regarding space charge profile of $5 \mathrm{wt} \%$ ASR, two different polarities of charge interact with each other during the whole measurement. Within initial 2 minutes, a huge amount of negative charges are accumulated in the bulk of specimen. When the measurement reaching 120 minutes, these negative charges become less and meanwhile the positive charges are gradually accumulated at the cathode, which indicates the occurrence of fast charge transport and recombination. For nanocomposites with lower loading ratios such as $0.5 \mathrm{wt} \%$ ASR, the space charge accumulation is effectively suppressed within 60 minutes in contrast to BPE specimen that shows fast accumulated heterocharges within first 2 minutes as shown in Fig. 4 (a1) and (b1). However, as the stressing time reaches 120 minutes, a few amount of space charges accumulate in the bulk of the specimen at $70 \mathrm{kV} / \mathrm{mm}$. To avoid the interference of capacitive charges and provide the profile of the injected charge, the measurement after removal voltage was conducted as illustrated in Fig. 4 (a2)-(c2). The injected heterocharges in the BPE can be observed as expected. With respect to 5 wt $\%$ ASR as shown in Fig. 4 (c2), the charge transportation is strongly intensified; the amount of injected charges in the $5 \mathrm{wt} \%$ ASR is much greater compared with BPE and $0.5 \mathrm{wt} \%$ ASR, and the charge decay in the $5 \mathrm{wt} \%$ ASR decreases most rapidly. Thus, the heterocharges owing to the transportation of charge carriers can be observed adjacent to the electrodes in the $5 \mathrm{wt} \%$ ASR. On the other hand, 
the charge transportation of $0.5 \mathrm{wt} \%$ ASR as shown in Fig. 4 (b2) is hindered even compared to the BPE; hence, only a small amount of charge close to the electrode can be observed after 2 minutes of removal voltage.
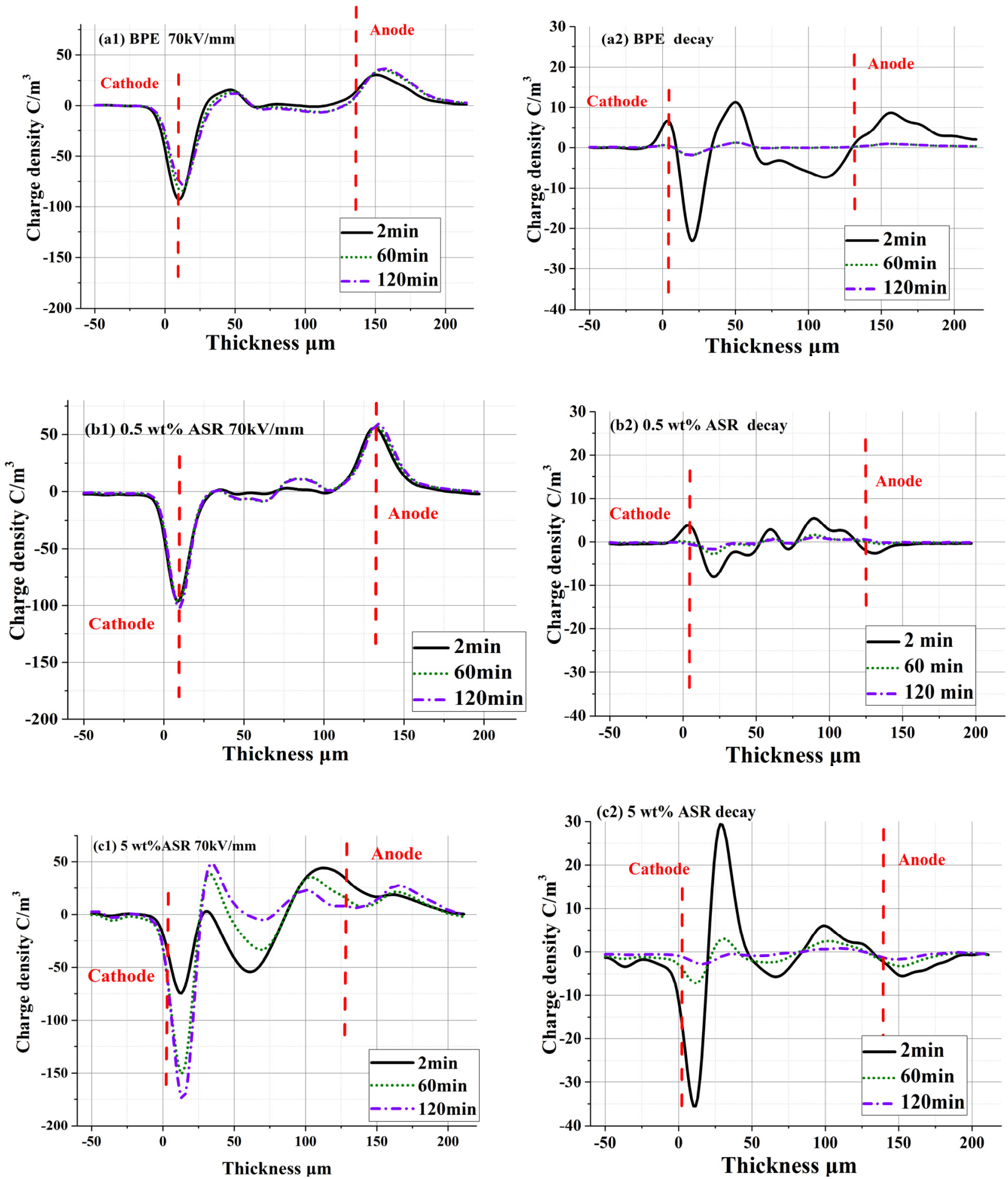

FIG. 4. Space charge profile stressed at 70kV/mm (a1) BPE, (b1) $0.5 \mathrm{wt} \%$ ASR, (c1) $5 \mathrm{wt} \%$ ASR; space charge profile after removal voltage (a2) BPE, (b2) 0.5 $\mathrm{wt} \%$, (c2) $5 \mathrm{wt} \%$ ASR 
To further validate the above results - i.e. that the heterocharges shown in the $5 \mathrm{wt} \%$ ASR are either transported from the other electrode or ionic charge carriers from the dissociation, and that the impact of loading ratio on the space charge can be either suppressed or facilitated - the PEA measurements were conducted with two configurations on double-layer specimens, including $5 \mathrm{wt} \%$ ASR and $0.5 \mathrm{wt} \%$ ASR. These two specimens are laminated as illustrated in Fig. 5, one is tightly placed on the surface of the other one without any additional binder. Since the thickness of laminated specimen was around $240 \mu \mathrm{m}$, which is too thick to apply $50 \mathrm{kV} / \mathrm{mm}$ for the specimen because the maximum voltage of the employed PEA system is $10 \mathrm{kV}$. Thus, the electric field applied for this measurement was reduced to $40 \mathrm{kV} / \mathrm{mm}$. The $0.5 \mathrm{wt} \%$ ASR layer hinders the charge injection while the $5 \mathrm{wt} \%$ ASR layer facilitates both positive and negative charge injection. Thus, two phenomena are expected depending on the position of the $5 \mathrm{wt} \%$ ASR layer. When the $5 \mathrm{wt} \%$ ASR layer is next to the anode, as illustrated in Fig. 5 (a), the negative charges are not able to inject from $0.5 \mathrm{wt} \%$ ASR, while a large amount of positive charges can easily inject from the $5 \mathrm{wt} \%$ ASR layer side. When these positive charges reach the interface between two layers, the $0.5 \mathrm{wt} \%$ ASR layer hinders the transport of these charges by suppressing injection; thus, the positive charges cannot further go through the $0.5 \mathrm{wt} \% \mathrm{ASR}$ layer to the cathode, and they will accumulate at the interface. As illustrated in Fig. 5 (b), placing the 5 wt $\%$ ASR layer next to the cathode can enhance negative charge injection and facilitate negative charge movement. On the other hand, the $0.5 \mathrm{wt} \%$ layer will hinder the positive charge injection from the anode and the negative charge injection from the $5 \mathrm{wt} \%$ ASR layer. Thus, the negative charge clearly accumulates at the interface, but few positive charges can be observed.

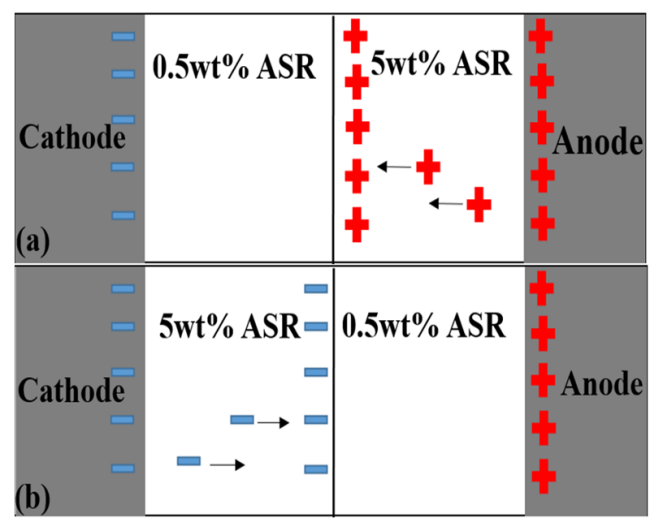

FIG. 5. The schematic diagram of the PEA system for two layer specimen (a) $5 \mathrm{wt} \%$ ASR layer next to the anode; (b) $5 \mathrm{wt} \%$ ASR layer next to the cathode The PEA results of the two-layer measurement are presented in Fig. 6. Positive charges in Fig. 6 (a) and negative charges in Fig. 6 (b) are observed at the interface between two layers, which is consistent with expectations. It is also observed that the charge formation at the interface between the two layers is extremely fast and reaches a quasi-steady state after 10 minutes of voltage application. This indicates that the observed heterocharges come from the opposite electrode due to facilitated charge transport in the $5 \mathrm{wt} \%$ ASR layer, and the injection of charge carriers can be effectively suppressed by the $0.5 \mathrm{wt} \%$ ASR layer. 

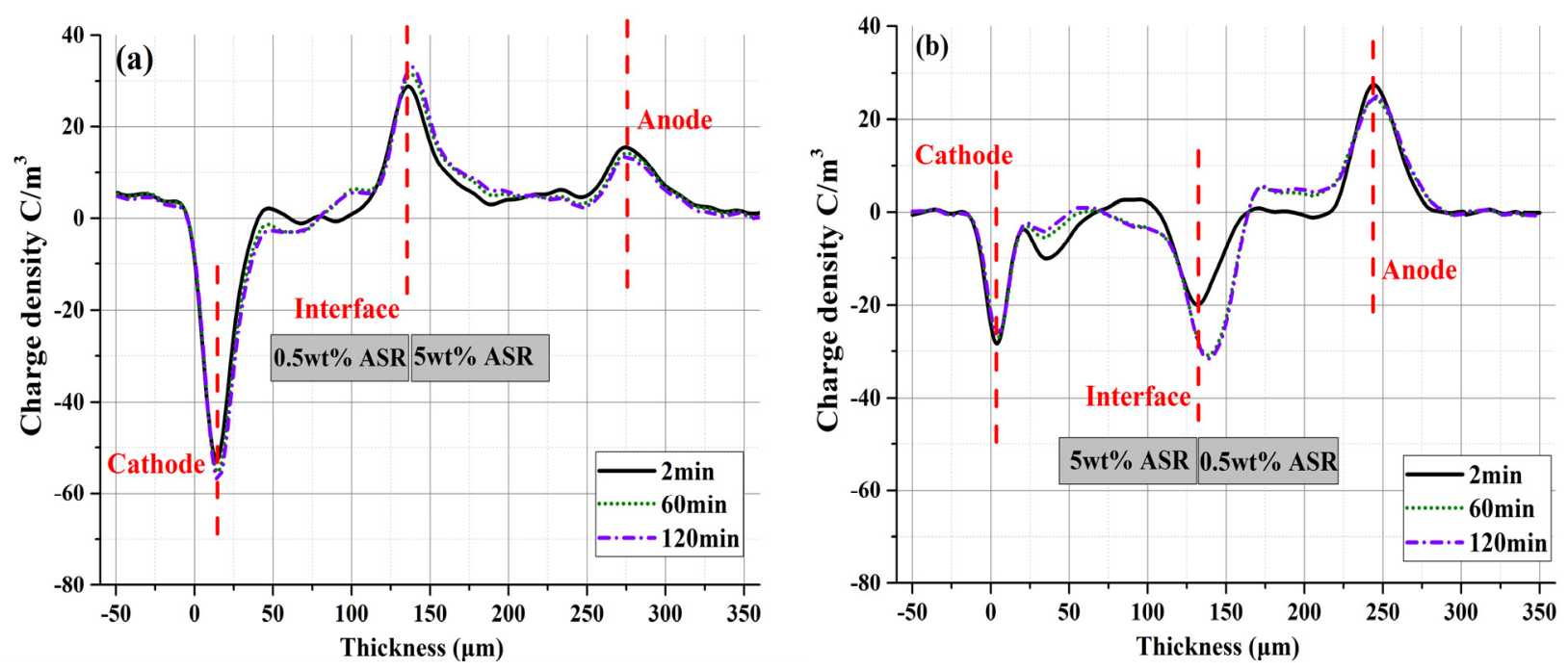

FIG. 6. The PEA measurement of the two layers specimen (a) 5wt $\%$ ASR layer connecting with anode; (b) $5 \mathrm{wt} \%$ ASR layer connecting with cathode

\section{B. ISPD}

All the potential curves presented in this study were conducted with over three times. Regarding the surface potential decay, a steady curve means that the traps in this material are deeper and the trapped charges are more difficult to escape in contrast to the rapidly dropped surface potential curve [3]. As illustrated in Fig. 7, the surface potential of $0.5 \mathrm{wt} \%$ ASR shows slowest decay, and the surface potential of $10 \mathrm{wt} \%$ ASR is the fastest. The decay rate of the BPE is comparable to both $2 \mathrm{wt} \%$ and 5 wt $\%$ ASR when the grid voltage setting at $4.8 \mathrm{kV}$. From Fig. 7 (b1) and (c1), the surface potential of $2 \mathrm{wt} \%$ and $5 \mathrm{wt} \%$ ASR specimens decays faster than that of the BPE as a consequence of the increasing grid voltage to $6 \mathrm{kV}$ and $7.2 \mathrm{kV}$. The initial potential of $10 \mathrm{wt} \%$ ASR is clearly lower than that of the other specimens. Although the system delay of the first data recorded may have impacted the value of the initial potential, the transportation of charge carriers during the corona charging is mainly responsible for the lower initial potential of $10 \mathrm{wt} \%$ ASR. The similar result according to surface potential decay of titanium dioxide/polyamide nanocomposites and thin film specimen was also reported [27,28]. The surface potential decay of BPE, 0.5 $\mathrm{wt} \%$ ASR and $5 \mathrm{wt} \%$ ASR at grid voltage $6 \mathrm{kV}$ and $7.2 \mathrm{kV}$ are in line with the results of space charge after removal voltage as illustrated in Fig. 4. Compared with BPE, the low loading ratios (0.5 wt $\%$ ASR) suppresses space charge accumulation and decays slowly while the high loading ratios ( $5 \mathrm{wt} \%$ ASR) enhances space charge accumulation and decays fast. Additionally, these observed phenomena are more obviously for specimen stressed at higher grid voltages. 

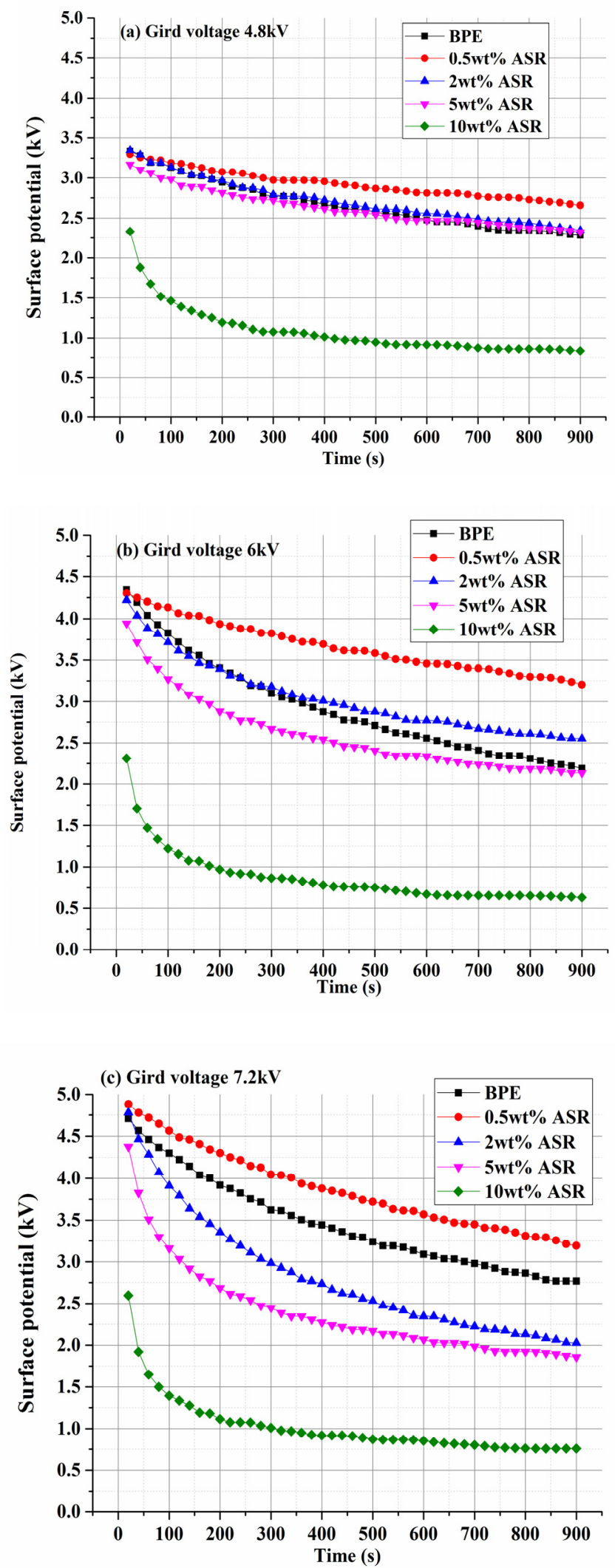

FIG. 7. Surface potential decay (a) ASR specimen with grid voltage $4.8 \mathrm{kV}$, (b) ASR specimen with grid voltage $6 \mathrm{kV}$, (c) ASR specimen with grid voltage 7.2kV 
The relationship between the average decay rate and surface potential is expressed as follows, where the D is the decay rate, $\mathrm{V}_{(\mathrm{t})}$ is the surface potential at $\mathrm{t}, \mathrm{V}_{(0)}$ is the first recorded surface potential.

$\mathrm{D}=\frac{\mathrm{V}_{(\mathrm{t})}-\mathrm{V}_{(0)}}{\mathrm{V}_{(0)}} \times 100 \%$

surface potential measurements can be used to find the trap distribution by analyzing its time derivative. Drawing the tdV/dt versus the energy of traps is a useful approach for finding trap distributions [33], because this method can be used without the a-priori knowledge on the trap parameters and overcome the small value of derivative $\mathrm{dV} / \mathrm{dt}$ at long time interval [34]. The energy of the traps is as function of time by considering the demarcation energy, $\mathrm{E}_{\mathrm{m}}$, which indicates the border between the emptied and occupied traps [33]. As the time increases, $E_{m}$ moves away from the conduction band and the relationship between the time and energy gap is defined as $[35,36]$.

$\mathrm{E}_{\mathrm{c}}-\mathrm{E}_{\mathrm{m}}=\mathrm{kT} \ln (\mathrm{vt})$

$\mathrm{t} \frac{\mathrm{dV}(\mathrm{t})}{\mathrm{dt}} \propto \mathrm{N}\left(\mathrm{E}_{\mathrm{m}}(\mathrm{t})\right) \frac{\mathrm{dE}_{\mathrm{m}}}{\mathrm{dt}}=\mathrm{N}\left(\mathrm{E}_{\mathrm{m}}(\mathrm{t})\right) \mathrm{kT} \frac{\mathrm{N}_{\mathrm{t}}}{\mathrm{N}_{\mathrm{c}}} \tau_{\mathrm{o}}$

Where $E_{c}$ is the conduction band energy level and $v$ is the attempt to escape frequency, which is set as $4.17 \times 10^{13} \mathrm{~s}^{-1}$ based on the work in [3]. The tdV/dt is proportional to the trap energy density $N\left(E_{m}\right)$ at the level $E_{m}(t)$. In Eq. $4, N_{t}$ and $N_{c}$ stand for trap and conduction states density respectively, $\tau_{\mathrm{o}}$ is the carrier lifetime in the conduction states, $\mathrm{k}$ is the Boltzmann's constant, and $\mathrm{T}$ is absolute temperature.

Fig. 8 (a) shows the decay rates of surface potential calculated according to Eq. 1, which reveals the impact of the grid voltage on the surface potential decay as a function of the nanosilica loading ratios. It can be noted that the decay rate of a specimen increases as the grid voltage increases. The decay rate decreases from $0 \mathrm{wt} \%$ to $0.5 \mathrm{wt} \%$, and then increases from 0.5 wt $\%$ to $10 \mathrm{wt} \%$ with different grid voltages. For the ASR specimen, the decay rate is larger than for the BPE starting from 2 $\mathrm{wt} \%$. The presence of deep trap in this nanocomposite is experimentally confirmed [23]. Comparing the trap distributions shown in Fig. 8 (b) and 8 (c), which are calculated based on the Eq. 2 and Eq. 3, the obtained results shows the profile of the distributions as a function of loading ratio and grid voltage. The maximum value located at a high energy level means that deep traps exist in the specimen, which can slow the mobility of trapped charge carriers [28]. After filling with the nanosilica, the trap depth moves to the higher energy level according to the maximum value appearing at energy $\sim 0.86 \mathrm{eV}$ with $0.5 \mathrm{wt} \% \mathrm{ASR}$, as shown in Fig. 8 (b). However, as the loading ratio increases, two peaks appear in the $5 \mathrm{wt} \%$ ASR, and its maximum value 
becomes lower at energy $\sim 0.85 \mathrm{eV}$, but it is still higher than the maximum value of BPE, which appears at $0.83 \mathrm{eV}$. Fig. 8(c) uses the $0.5 \mathrm{wt} \%$ ASR as an example to show the impact of increasing the electric field on the trap distribution. It is evident that the maximum value in trap depth decreases as the grid voltage increases. The amount of deep traps is theoretically proportional to the nanosilica loading ratios. Hence, the nanocomposites with high loading ratios should slow the surface potential decay more effectively than the nanocomposites with low loading ratios. However, based on the results in this experiment, $0.5 \mathrm{wt} \%$ ASR clearly reduce the decay rate compared with the BPE and other nanocomposites. From the perspective of energy distribution, deep traps can obstruct the mobility of trapped charge carriers and show stable surface potential decay, but the observed results does not show this function in $5 \mathrm{wt} \%$ ASR, which even has more deep traps in contrast to $0.5 \mathrm{wt} \%$ ASR. In addition to the loading ratios, the results obtained from nanocomposites measurement especially for the one loaded with more nanosilica are more significantly affected by electric fields in contrast to the BPE specimen.
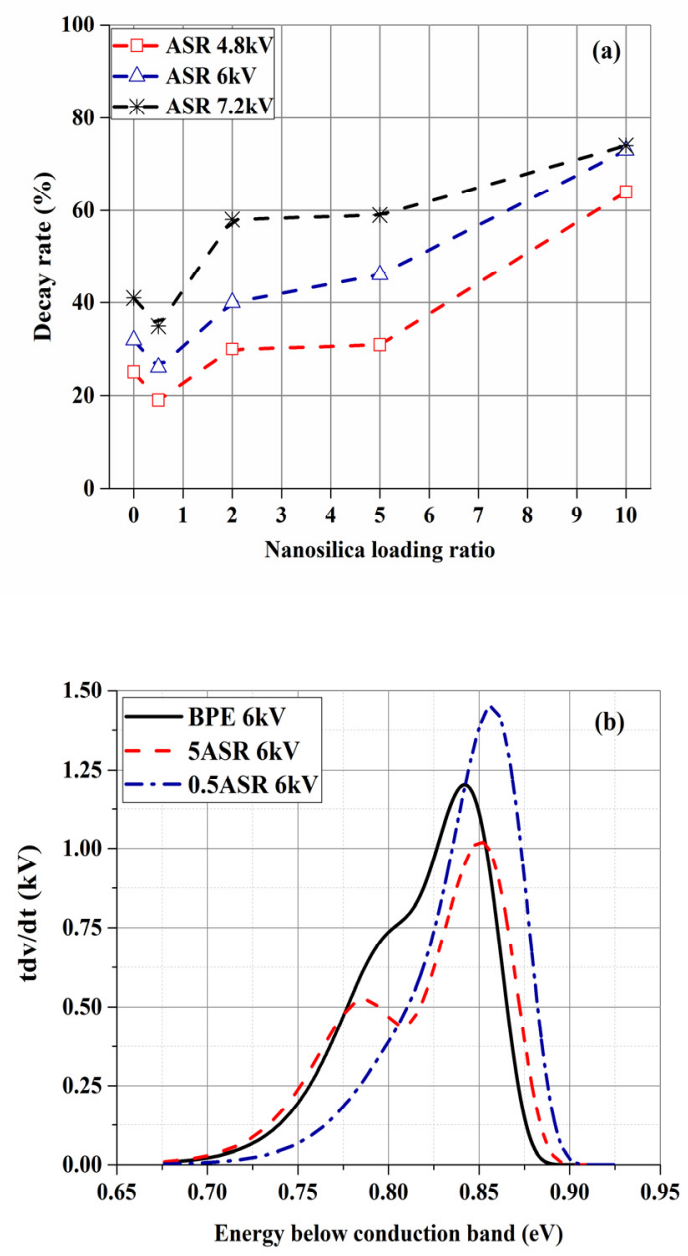


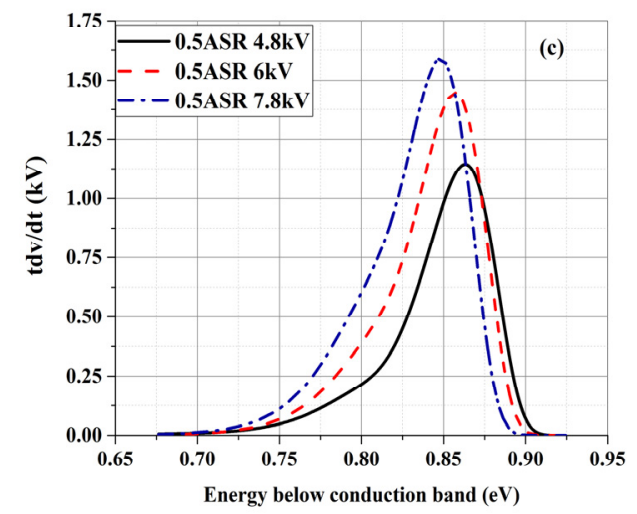

FIG. 8 Analysis of surface potential decay (a) the relationship between the decay rate and the loading ratios with different grid voltages; (b) the trap distribution at $6 \mathrm{kV}$ varying with the loading ratios; (c) trap distribution of $0.5 \mathrm{wt} \%$ ASR with different grid voltage;

\section{DISCUSSION WITH TUNNELING MODEL}

Trap sites have already been employed for investigating the charge dynamics for conventional polymer dielectrics. The amorphous domains and the chemical defects of conventional polyethylene create the trap sites, which can be used by charge carriers to hop over the energy barriers or tunneling between the two adjacent trap sites in the case of shorter distance. Therefore, both the hopping process and the tunneling process can account for the bulk conduction of the polymer. The hopping mechanism is usually used to explain the charge transport in the neat LDPE [18], which possesses fewer and shallower traps compared with the nanocomposites. Based on the space charge behaviour in nanodielectrics, Chen et al. suggested the tunnelling process as an explanation for the observed charge behaviour in nanodielectrics with high loading ratios [16]. This model offers a good explanation on space charge suppression of nanodielectrics with low loading ratios and more complicated charge dynamics of nanodielectrics with high loading ratios by considering the impact of deep traps and the tunneling process. For nanodielectrics with high loading ratios where deep traps have been introduced, the estimated charge transport characteristics at constant temperature can be simply represented as Eq.4 [16]:

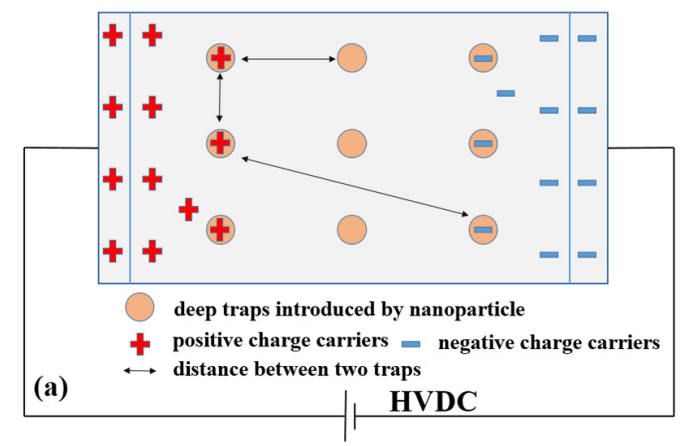




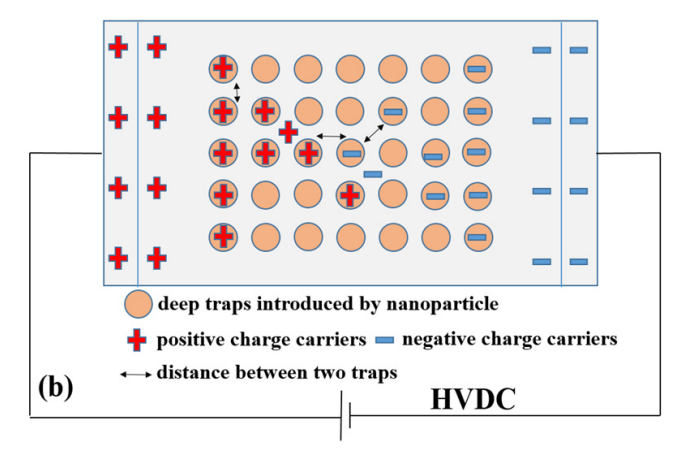

FIG. 9. Schematic model showing uniform charge distribution in nanodielectrics with the (a) low loading ratio and (b) high loading ratio

$\mathrm{J}=\frac{\mathrm{CE}^{2}}{\mathrm{E}_{\mathrm{t}}} \exp \left(-\frac{B E_{\mathrm{t}}}{\mathrm{E}}\right) \exp \left(-\frac{2 \mathrm{~d}}{\mathrm{~d}_{0}}\right)$

Where $\mathrm{C}$ and $\mathrm{B}$ are constants, $\mathrm{E}$ is the electric field, $\mathrm{E}_{\mathrm{t}}$ is the trap depth or potential barrier height, $\mathrm{d}$ is the separation distance between the two adjacent particles in the field direction, $\mathrm{d}_{0}$ is the characteristic tunneling distance.

The bipolar charge injection is considered after the electric field exceeding the threshold value [16,17,37]. According to the Eq. 4, the electric field is an important factor that further affects the tunnelling current. For the same distance between two adjacent deep traps, applying a higher electric field promotes the tunnelling process. According to the bipolar charge injection and recombination occurring in bulk, the electric field exerts the transportation of charge carriers. Compared to the BPE specimens, the deep traps introduced by the nanoparticles capture the charge carriers in the sub-surface region. These trapped charges generate an opposite electric field to the applied electric field. Therefore, the electric field at the interface of specimen and electrode is reduced, which hinders further charge injection. For the nanocomposites with low loading ratios, as shown in Fig. 9 (a), the separation distance between two adjacent traps is large, and the captured charges may not have sufficient energy to escape from the traps. Thus, the charge carriers are difficult to move through the bulk of nanocomposites and the stable charge can be observed. Conversely, for higher loading ratio nanocomposites, higher than $5 \mathrm{wt} \%$ as results presented in this research and similar work reported in $[38,39]$, the short separation distance due to the increased number of deep traps as shown in Fig. 9 (b) prompts the tunnelling process to dominate the transport of charge carriers to the adjacent traps in contrast to overcoming the energy barrier [26]. Consequently, the captured charges are able to escape from the traps located near the subsurface and continue to transport through the bulk, and charge carriers can continue to be injected into the nanocomposites as opposite field at the interface becomes low. In addition, the tunnelling process can facilitate the charge recombination between two traps that capture the opposite polarity of charges. The recombination process causes more charge carrier injections and 
facilitates the charge transport. Hence, the observed phenomena of charge dynamics in nanodielectrics with high loading ratios tend to be more complicated and intensified than in those with low loading ratios [16].

Based on the description above, the tunnelling process can effectively explain the results obtained by these measurement. Regarding the space charge profile of $5 \mathrm{wt} \%$ ASR, $50 \mathrm{kV} / \mathrm{mm}$ is sufficient to make the tunnelling process occur as a result of the large amount of deep traps and the short distance between them. However, with respect to the $0.5 \mathrm{wt} \% \mathrm{ASR}$, even $70 \mathrm{kV} / \mathrm{mm}$ is still too low to facilitate the charge transport as $5 \mathrm{wt} \%$ ASR does because the amount of deep traps is small, and the distance between traps is thereby large correspondingly, which is not able to cause tunnelling process to take place. Therefore, the space charge accumulation and transportation of $0.5 \mathrm{wt} \%$ ASR is not obvious because deep traps can effectively reduce the mobility of charge carriers. As the external electric field is removed, the tunnelling process still affects the abundant charges due to the high internal electric field, which will dominate the charge dynamics in the initial decay period and result in the fast space charge decay for nanocomposites with high loading ratios, such as $5 \mathrm{wt} \% \mathrm{ASR}$. In the case of isothermal surface potential decay, since $0.5 \mathrm{wt} \%$ ASR has more deep traps than BPE due to the incorporated nanosilica, many charges are captured by these deep traps and remain stable. Moreover, in the $0.5 \mathrm{wt} \%$ nanocomposites the long distance between adjacent traps associated with the applied initial surface potential, which is related to the electric field, prevents the tunnelling process from happening. In the case of nanocomposites with high loading ratios, the distance is crucial owing to the increased number of deep traps, as mentioned in the description of the tunnelling model. For instance, the $10 \mathrm{wt} \%$ ASR has a shorter distance between traps than the ASR nanocomposites with $0.5 \mathrm{wt} \%, 2 \mathrm{wt} \%$, and $5 \mathrm{wt} \%$. The shorter distance and the electric field established by the deposited charge are capable of starting the tunnelling process, which dominates the charge transport in the $10 \mathrm{wt} \% \mathrm{ASR}$. Therefore, the fastest decay rate can be observed in this specimen. Based on the tunnelling model, the electric field is important to the charge dynamics in nanodielectrics. The grid voltage is raised in the ISPD experiment, but the thickness of the specimen remains consistent. Therefore, the grid voltage can also indicate the effect of the electric field to some extent. The decay rate of all the specimens increases with the level of the initial potential growth, which is also called the crossover phenomenon caused by charge recombination[17,40]. According to the tunnelling model, the high electric field can facilitate the charge transport by the tunnelling process in nanocomposites. The specimen containing more nanoparticles is much more easily affected by the increased electric field. For example, the decay of $2 \mathrm{wt} \%$ ASR and $5 \mathrm{wt} \%$ ASR decreases dramatically compared with BPE when the grid voltage applied increasing. As for $10 \mathrm{wt} \% \mathrm{ASR}$, the amount of deep traps in this specimen is sufficient to cause tunnelling process to happen at $3.6 \mathrm{kV}$ grid voltage during the corona charging, and its decay rate is nearly approach of the maximum value, which is tunnelling process can provide based on this loading ratio. Therefore, the initial potential and 
decay rate do not obviously change as the voltage increases. The method tdV/dt is frequently used for analysis the surface potential decay results. However, the difference of trap depth obtained among $0.5 \mathrm{wt} \%, 5 \mathrm{wt} \%$ and BPE is too small as presented in the Fig. 8. Since the nanosilica introduce the deep traps in the system, the tunnelling may also occur during the measurement which should affect the obtained results. The gird voltage and the nanosilica loading ratios affect the process of decay. Hence, this method can only be used to analyze the surface potential decay qualitatively rather than offering an accurate trap depth. Moreover, since the presence of deep trap in the nanocomposites is experimentally confirmed [23], the tunnelling process employed with deep traps is considered as a main approach of charge transport in the nanocomposites rather than hopping process that mainly employs shallow traps. Although it is difficult to experimentally validate the tunnelling process occurring in the nanocomposites, the temperature dependent DC conductivity results of the nanocomposites has been processed for the other publication, which will reveal more information related the mechanism of charge transport in the nanocomposites.

\section{CONCLUSION}

To conclude this paper, the tunneling model can effectively explain the experiment results obtained from isothermal surface potential decay and space charge measurement, which highlight the effect of nanoparticle loading ratios and the electric fields on the charge dynamics in the nanocomposites. The main conclusions may be drawn as follows.

1. The results of isothermal surface potential and space charge are consistent with each other. For the nanocomposites incorporated with untreated nanosilica, the low loading ratios such as $0.5 \mathrm{wt} \%$ hinder the charge dynamics including space charge accumulation and charge transportation whereas the high loading ratios including $2 \mathrm{wt} \%, 5 \mathrm{wt} \%$ and $10 \mathrm{wt} \%$ will facilitate the charge dynamics in contrast to the unfilled blend polyethylene.

2. The heterocharges observed in the nanocomposites with high loading ratios are transported from the other electrode.

3. By employing the tunneling model, the space charge accumulation and fast charge transport of nanocomposites with high loading ratios and stressed at high electric fields, can be explained effectively and reasonably. Therefore, the tunneling process is suggested as a mechanism of charge transport in the nanocomposites, which is worth being further validated and considered.

\section{REFERENCES}

[1]Kirby N, Horwill C, and MacLeod N Year 2012 IEEE Power and Energy Society General Meeting. [2]Fothergill J C Year 2014 IEEE Electrical Insulation Conference (EIC).

[3]Shen W-W, Mu H-B, Zhang G-J, Deng J-B, and Tu D-M 2013 Journal of Applied Physics 113 (8), 083706 . 
[4]Lewis T 1990 Journal of Physics D: Applied Physics 23 (12), 1469.

[5]Lewis T 1994 IEEE Transactions on Dielectrics and Electrical Insulation 1 (5), 812.

[6]Roy M, Nelson J K, MacCrone R, and Schadler L 2007 Journal of Materials Science 42 (11), 3789.

[7]Murakami Y, Nemoto M, Okuzumi S, Masuda S, Nagao M, Hozumi N, Sekiguchi Y, and Murata Y 2008 IEEE Transactions on Dielectrics and Electrical Insulation 15 (1).

[8]Hosier I, Praeger M, Vaughan A, and Swingler S Year 2015 IEEE Electrical Insulation Conference $(E I C)$.

[9]Yan W, Chen G, and Vaughan A Year 2014 IEEE Conference on Electrical Insulation and Dielectric Phenomena (CEIDP).

[10]Lau K, Vaughan A, Chen G, Hosier I, Holt A, and Ching K Y 2014 IEEE Transactions on Dielectrics and Electrical Insulation 21 (1), 340.

[11]Qiang D, He M, Chen G, and Andritsch T Year 2015 IEEE Electrical Insulation Conference (EIC).

[12]Takada T, Hayase Y, Tanaka Y, and Okamoto T 2008 IEEE Transactions on Dielectrics and Electrical Insulation 15 (1).

[13]Nelson J K, Fothergill J C, Dissado L A, and Peasgood W 2002.

[14]Lewis T 2004 IEEE Transactions on Dielectrics and Electrical Insulation 11 (5), 739.

[15]Tanaka T, Kozako M, Fuse N, and Ohki Y 2005 IEEE Transactions on Dielectrics and Electrical Insulation 12 (4), 669.

[16]Chen G, Li S, and Zhong L Year 2015 IEEE 11th International Conference on the Properties and Applications of Dielectric Materials (ICPADM).

[17]Chen G 2010 Journal of Physics D: Applied Physics 43 (5), 055405.

[18]Brunson J 2010.

[19]Tu D M, Liu W B, Zhuang G P, Liu Z Y, and Kao K C 1989 IEEE Transactions on Electrical Insulation 24 (4), 581.

[20]Chen G and Xu Z 2009 Journal of Applied Physics 106 (12), 123707.

[21]Han B, Wang X, Sun Z, Yang J, and Lei Q 2013 Applied physics letters 102 (1), 012902.

[22]Huang X, Jiang P, and Yin Y 2009 Applied Physics Letters 95 (24), 242905.

[23]Wang Y, Hao M, Xu Z, Qiang D, Chen G, and Vaughan A 2018 Applied Physics Letters 113 (2), 022904.

[24]Yan W, Zhiqiang X, George C, and Vaughan A Year Electrical Insulation and Dielectric Phenomena (CEIDP), 2015 IEEE Conference on.

[25]Lau K, Vaughan A, Chen G, and Hosier I Year Journal of Physics: Conference Series.

[26]Li G, Chen G, and Li S 2016 Applied Physics Letters 109 (6), 062901.

[27]Zha J, Chen G, Dang Z, and Yin Y 2011 Journal of Electrostatics 69 (3), 255.

[28]Xu Z, Zhang L, and Chen G 2007 Journal of Physics D: Applied Physics 40 (22), 7085.

[29]Chen G, Xu Z, and Zhang L 2007 Measurement Science and Technology 18 (5), 1453.

[30]Wang Y, Xu Z, Chen G, and Vaughan A Year 2016 IEEE International Conference on Dielectrics (ICD).

[31]Lau K Y, University of Southampton, 2013.

[32]Chen G, Tay T Y G, Davies A E, Tanaka Y, and Takada T 2001 IEEE Transactions on Dielectrics and Electrical Insulation 8 (6), 867.

[33]Molinié P 2005 IEEE Transactions on Dielectrics and Electrical Insulation 12 (5), 939.

[34]Sjostedt H, Gubanski S M, and Serdyuk Y V 2009 IEEE Transactions on Dielectrics and Electrical Insulation 16 (3), 696.

[35]Simmons J and Tam M 1973 Physical Review B 7 (8), 3706.

[36]Watson P K 1990 Journal of Physics D: Applied Physics 23 (12), 1479.

[37]Liu N, Zhou C, Chen G, and Zhong L 2015 Applied physics letters 106 (19), 192901. 
[38]Green C and Vaughan A 2008 IEEE Electrical Insulation Magazine 24 (4), 6.

[39]Hosier I, Praeger M, Holt A, Vaughan A, and Swingler S Year Electrical Insulation and Dielectric Phenomena (CEIDP), 2014 IEEE Conference on.

[40]Ieda M, Sawa G, and Shinohara U 1967 Japanese Journal of Applied Physics 6 (6), 793. 